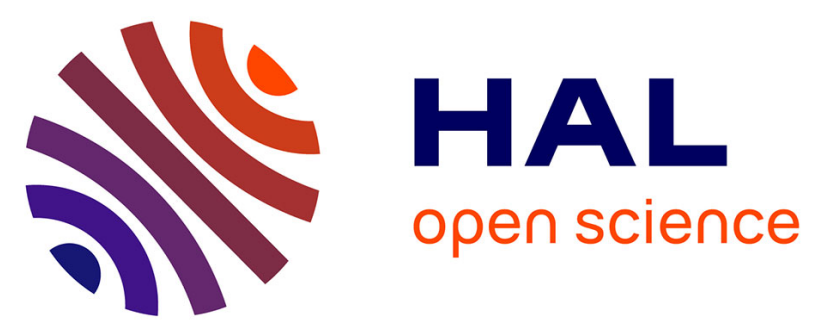

\title{
Influence de traitements magnétothermiques sur les performances magnétiques d'alliages amorphes à base cobalt. Application aux têtes magnétiques
}

R. Barrue, J.C. Perron, J. Robert

\section{- To cite this version:}

R. Barrue, J.C. Perron, J. Robert. Influence de traitements magnétothermiques sur les performances magnétiques d'alliages amorphes à base cobalt. Application aux têtes magnétiques. Revue de Physique Appliquée, 1987, 22 (6), pp.399-405. 10.1051/rphysap:01987002206039900 . jpa-00245554

HAL Id: jpa-00245554

https://hal.science/jpa-00245554

Submitted on 1 Jan 1987

HAL is a multi-disciplinary open access archive for the deposit and dissemination of scientific research documents, whether they are published or not. The documents may come from teaching and research institutions in France or abroad, or from public or private research centers.
L'archive ouverte pluridisciplinaire HAL, est destinée au dépôt et à la diffusion de documents scientifiques de niveau recherche, publiés ou non, émanant des établissements d'enseignement et de recherche français ou étrangers, des laboratoires publics ou privés. 
Classification

Physics Abstracts

75.60

\title{
Influence de traitements magnétothermiques sur les performances magnétiques d'alliages amorphes à base cobalt. Application aux têtes magnétiques
}

\author{
R. Barrue, J. C. Perron et J. Robert \\ Laboratoire de Génie Electrique des Universités Paris VI et Paris XI (LGEP-ESE), associé au CNRS N 127 , \\ plateau du Moulon, 91190 Gif-sur-Yvette, France
}

(Reçu le 17 novembre 1986, accepté le 2 mars 1987)

\begin{abstract}
Résumé. - Les rubans ferromagnétiques amorphes paraissent a priori posséder la plupart des propriétés exigées des matériaux constitutifs de têtes magnétiques, la nécessité de s'affranchir de la magnétostriction privilégiant les nuances au cobalt. Deux alliages de ce type, produits industriellement, ont été testés dans la bande de fréquences $50 \mathrm{~Hz}-50 \mathrm{kHz}$, après avoir été soumis à des traitements thermiques ou magnétothermiques. L'influence de ces traitements sur les pertes et la perméabilité est examinée. Il apparaît que l'une des nuances (appellation commerciale $2705 \mathrm{M}$ ), présente des performances intéressantes pour l'application envisagée. Ces performances sont obtenues sur un tore constitué, soit de rondelles découpées dans le ruban, soit de ruban enroulé sur lui-même, le champ étant appliqué normalement aux faces du tore. La visualisation de la structure en domaines conduit à une interprétation qualitative des mesures.
\end{abstract}

\begin{abstract}
Amorphous ferromagnetic ribbons seem to possess most of the properties required for magnetic head materials. The necessity of avoiding magnetostriction effect leads to use cobalt alloys. Two kinds of marketed cobalt alloys were annealed with or without magnetic field ; then, they were tested in the frequency range $50 \mathrm{~Hz}$ to $50 \mathrm{kHz}$. Influence of these treatments on losses and permeability is discussed. One of these two alloys (trade reference $2705 \mathrm{M}$ ) shows attractive performances for the expected application. These performances are achieved with a torus made of rings cut in the ribbon, or a rolled ribbon, and a magnetic field perpendicular to the torus sides. Visualization of the domain structure leads to a qualitative interpretation of the measures.
\end{abstract}

\section{Introduction.}

L'enregistrement magnétique a fait ces dernières années des progrès sensibles. Le ferrite doux, de résistivité élevée, demeure aux fréquences supérieures à $10 \mathrm{kHz}$, le matériau le plus fréquemment utilisé pour la fabrication des têtes.

Idéalement, un matériau constitutif de tête doit présenter une induction à saturation et une résistivité élevées. Sa perméabilité initiale doit être grandé pour les têtes de lecture, sa perméabilité sous champ élevé, grande, pour les têtes d'impression. A ce jour, aucun matériau susceptible d'être utilisé industriellement ne possède l'ensemble de ces qualités [13]. Aussi divers auteurs pensent-ils que les alliages ferromagnétiques amorphes représentent une voie nouvelle $[1,4,5]$, d'autant plus que leur dureté $\left.{ }^{1}\right)$ constitue également un élément favorable.

(1) Typiquement 1000 pour la dureté Vickers $\left(\mathrm{HV}_{0,2}\right)$.
L'objet du présent article est de décrire l'influence de traitements thermiques ou magnétothermiques sur les performances magnétiques d'alliages amorphes, a priori bien adaptés à la réalisation de têtes magnétiques destinées à l'enregistrement des sons (têtes « audio »).

\section{Dispositif expérimental.}

Seul l'emploi d'alliages amorphes au cobalt peut à ce jour être raisonnablement envisagé. En effet, l'usinage et le montage des têtes, nécessairement effectués après traitement thermique, impliquent une magnétostriction quasi nulle [6-8]. Nous avons expérimenté deux nuances largement commercialisées dont les caractéristiques principales sont précisées, tableau I.

Nous appliquons le(s) traitement(s) et en apprécions les effets sur des tores réalisés à l'aide du ruban 
Tableau I. - Caractéristiques principales des alliages amorphes au cobalt étudiés.

[Main characteristics of tosted Co-basis amorphous alloys.]

\begin{tabular}{c|c|c|c|c|c|c|c}
$\begin{array}{c}\text { Dénomination } \\
\text { commerciale }\end{array}$ & Fabricant & $\begin{array}{c}\text { Composition } \\
(\% \text { atomiques })\end{array}$ & $\begin{array}{c}\text { Induct. à } \\
\text { saturat. } \\
(\mathrm{T})\end{array}$ & $\begin{array}{c}\text { Temp. } \\
\text { Curie } \\
\left({ }^{\circ} \mathrm{C}\right)\end{array}$ & $\begin{array}{c}\text { Temp. } \\
\text { crist. } \\
\left({ }^{\circ} \mathrm{C}\right)\end{array}$ & $\begin{array}{c}\text { Coefficient } \\
\text { magnétostr. } \\
\left(\times 10^{6}\right)\end{array}$ & $\begin{array}{c}\text { Densité } \\
\left(\mathrm{g} . \mathrm{cm}^{-3}\right)\end{array}$ \\
\hline $\begin{array}{c}6025 \\
(\text { Vitrovac) }\end{array}$ & $\begin{array}{c}\text { Vacuumschmelze } \\
\text { (RFA) }\end{array}$ & $\mathrm{Co}_{66} \mathrm{Fe}_{4} \mathrm{Mo}_{2} \mathrm{Si}_{16} \mathrm{~B}_{12}$ & 0,55 & 250 & $\sim 500$ & $\leqslant 0,5$ & 7,7 \\
\hline $\begin{array}{c}2705 \mathrm{M} \\
(\text { Metglas) }\end{array}$ & $\begin{array}{c}\text { Allied Chemical } \\
\text { corporation } \\
\text { (USA) }\end{array}$ & $\begin{array}{c}\text { non révélée par le fabri- } \\
\text { cant }\end{array}$ & 0,7 & 330 & 520 & 0,5 & 8,0
\end{tabular}

à étudier. Nous avons mis en œuvre deux géométries, respectivement :

- « en spirale ». Cette dernière est constituée par le ruban enroulé sur un support,

- « en rondelles ». Celles-ci sont découpées mécaniquement dans le ruban et empilées sur un support.

Nous caractérisons un traitement thermique, ou recuit, par la température et la durée du (des) palier(s). Le champ magnétique éventuellement appliqué durant un recuit peut être (Fig. 1) :

- orienté selon la ligne moyenne du tore ; celle-ci se confond avec l'axe du ruban pour la géométrie en spirale. Nous estimons que le circuit magnétique présente des entrefers négligeables, ce qui permet de déterminer simplement le champ d'excitation. Nous parlons dans ce cas de recuit avec champ circonférentiel (RACC),

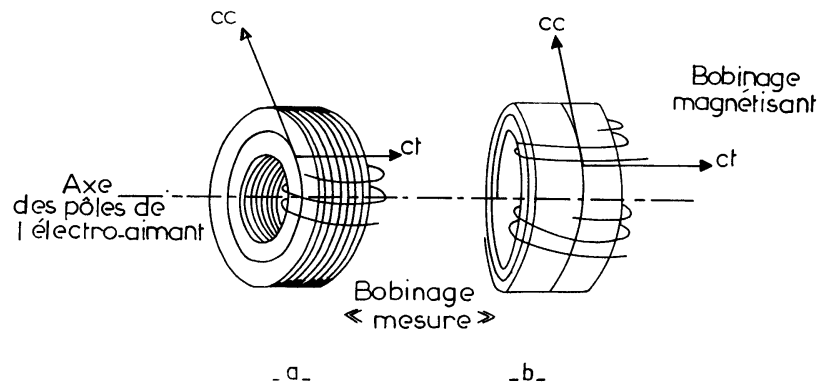

Fig. 1. - Dispositif expérimental. a - géométrie en rondelles. $\mathrm{b}$ - géométrie en spirale.

[Experimental apparatus. a - ring geometry. b-spiral geometry.]

- normal aux faces planes du tore. Pour ce faire, le four de traitement est placé entre les pôles d'un électro-aimant. Le champ démagnétisant au sein du matériau est difficile à évaluer. Aussi repérons-nous le champ résultant par la valeur de l'induction dans l'air. Nous parlons dans ce cas de recuit avec champ transverse (RACT) ${ }^{1}$ ).

${ }^{1}$ ) Les traitements thermiques purs sont désignés par le sigle RSC (recuit sans champ).
Les mesures sont effectuées après traitement en utilisant le bobinage porté par le tore. Le champ de mesure est donc circonférentiel.

Nous avons observé sur les deux nuances 6025 et $2705 \mathrm{M}$ le phénomène suivant, cité par ailleurs [1]. Un recuit sous champ circonférentiel conduit à des cycles d'hystérésis dissymétriques dès que la température de palier est supérieure à une certaine valeur $\left(270{ }^{\circ} \mathrm{C}\right.$ pour la nuance 6025$)$. Un exemple est donné, figure 2 . Nous pensons qu'il s'agit de thermorémanence. De l'oxyde de cobalt ou une phase de cobalt serait aimanté par le champ appliqué pendant le traitement, le champ coercitif étant suffisamment élevé pour que le champ alternatif de mesure soit sans effet. On inverse la dissymétrie en soumettant l'échantillon à un champ convenablement orienté et de valeur élevée. Pour ce faire, nous envoyons une impulsion de courant dans l'enroulement. Le champ, de l'ordre de $20000 \mathrm{~A} . \mathrm{m}^{-1}$, fait basculer le cycle d'hystérésis (Fig. 3). Pour éviter ce phénomène, nous avons mis en œuvre les traitements suivants, définis à partir d'essais préliminaires.

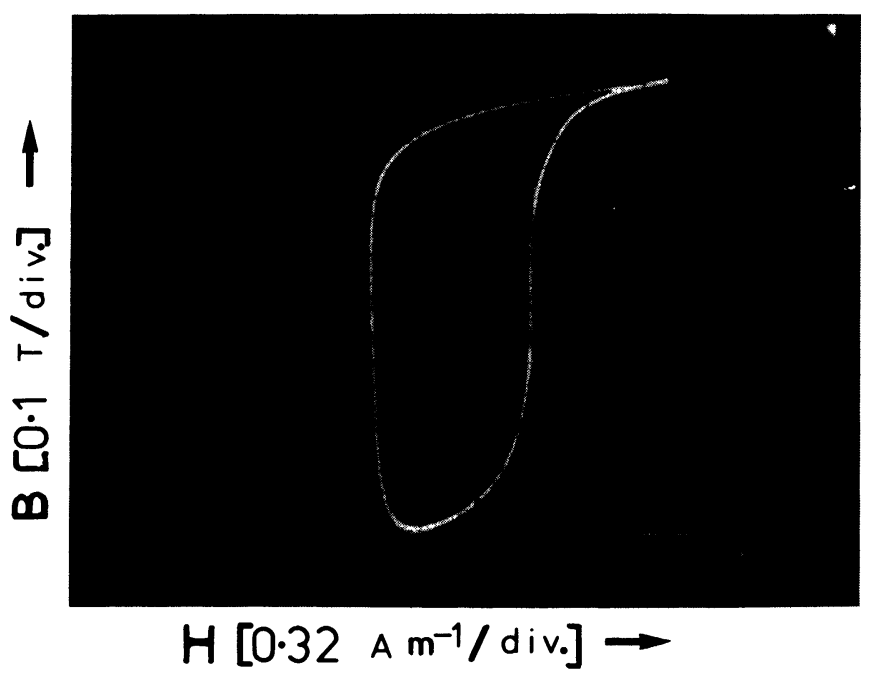

Fig. 2. - Cycle d'hystérésis dissymétrique. - Alliage 6025 ; - RACC $430{ }^{\circ} \mathrm{C}-1$ h-1 200 A.m ${ }^{-1}$; - Conditions de la mesure : $50 \mathrm{~Hz}-0,3 \mathrm{~T}$.

[Asymmetrical hysteresis loop. - Alloy 6025; - Circumferencial Field Annealing : $430{ }^{\circ} \mathrm{C}-1 \mathrm{~h}$ 1200 A.m ${ }^{-1}$; - Measure conditions : $50 \mathrm{~Hz}-0.3$ T.] 

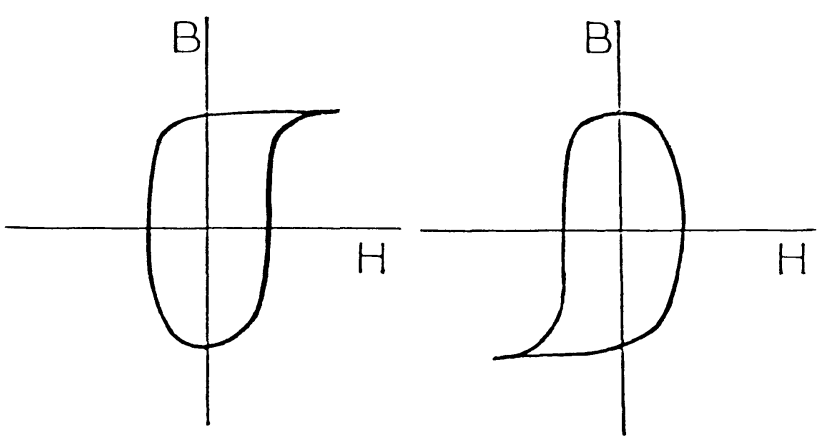

Fig. 3. - Basculement d'un cycle d'hystérésis sous l'influence d'un champ appliqué $\left(20000 \mathrm{~A} \cdot \mathrm{m}^{-1}\right)$.

[Rotation of an hysteresis loop induced by an external field $\left(20000\right.$ A.m $\left.{ }^{-1}\right)$.]

Tableau II. - Traitements magnétothermiques mis en oeuvre.

[Magnetothermal treatments.]

\begin{tabular}{|c|c|c|c|c|}
\hline \multicolumn{2}{|c|}{$\begin{array}{l}\text { Nature du } \\
\text { traitement }\end{array}$} & $\begin{array}{l}\text { Température } \\
\left({ }^{\circ} \mathrm{C}\right)\end{array}$ & $\begin{array}{l}\text { Durée } \\
\text { (h) }\end{array}$ & $\begin{array}{l}\text { Champ } \\
\text { appliqué }\end{array}$ \\
\hline \multicolumn{2}{|c|}{ RSC } & 430 & 1 & \\
\hline \multirow[b]{2}{*}{ RACC } & $1^{\mathrm{er}}$ palier & \multicolumn{3}{|c|}{ RSC } \\
\hline & $2^{\mathrm{e}}$ palier & $\begin{array}{l}240(6025) \\
315(2705 \mathrm{M})\end{array}$ & 1 & $1200 \mathrm{~A} \cdot \mathrm{m}^{-1}$ \\
\hline \multirow[b]{2}{*}{ RACT } & 1 er palier & \multicolumn{3}{|c|}{ RSC } \\
\hline & $2^{\mathrm{e}}$ palier & $\begin{array}{l}240(6025) \\
315(2705 \mathrm{M})\end{array}$ & 1 & $0,3 \mathrm{~T}$ \\
\hline
\end{tabular}

Dans ces conditions, la relaxation des contraintes a lieu essentiellement pendant le premier palier et la magnétisation du matériau pendant le second, à température suffisamment basse pour que le phénomène de thermorémanence n'apparaisse pas.

\section{Résultats expérimentaux.}

L'existence de deux alliages, de deux géométries, de trois types de traitements, et la nécessité d'examiner les dépendances en fréquence ont conduit à un volume important de résultats qui ne sauraient tous être examinés. Aussi avons-nous défini des règles de sélection :

- La géométrie en rondelles est bien adaptée à la réalisation de têtes magnétiques. Il ne faut pas néanmoins exclure celle en spirale parce qu'il est techniquement possible de noyer le tore dans une résine durcissable puis d'effectuer des usinages.

- Les caractéristiques doivent être linéaires dans la bande de fréquences «audio » considérée.

- Enfin, les pertes sont, à un moindre titre, un élément de comparaison.

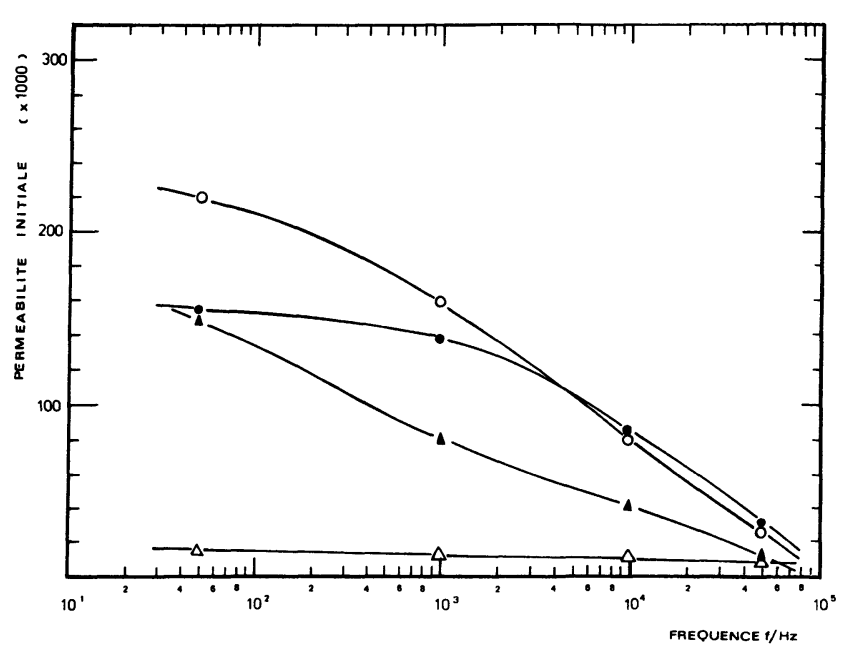

Fig. 4. - Dépendance en fréquence de la perméabilité initiale. - Alliage 6025-Géométrie en spirale ; - RSC, $\triangle$ RACC, O RACT, $\triangle$ Brut de livraison.

[Initial permeability as a function of frequency. - Alloy 6025-Spiral geometry; - Without Field Annealing, $\Delta$ Circumferential Field Annealing, $O$ Transversal Field Annealing, $\triangle$ As cast.]

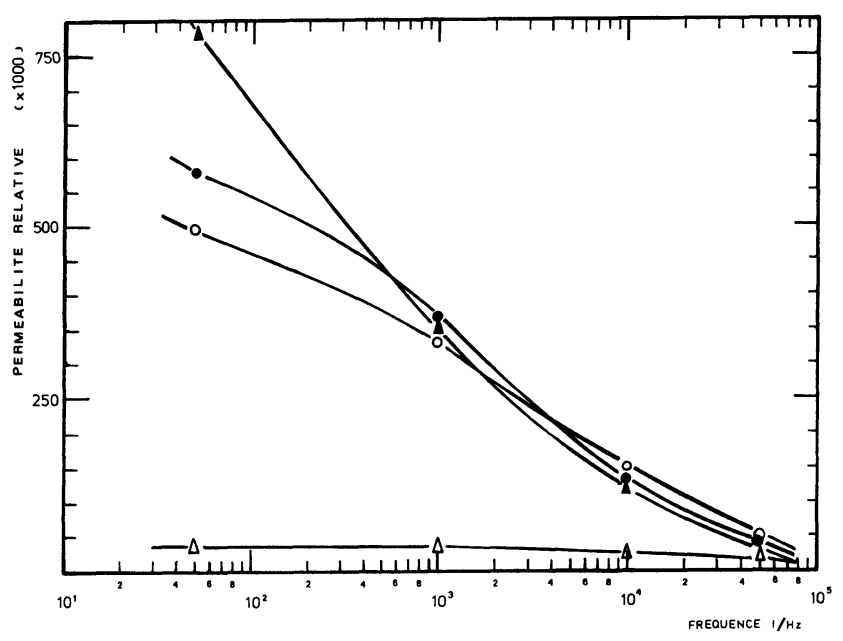

Fig. 5. - Dépendance en fréquence de la perméabilité relative maximum. - Alliage 6025-Géométrie en rondelles (mêmes symboles que sur la Fig. 4).

[Maximum relative permeability as a function of frequency. - Alloy 6025-Ring geometry (symbols are those of Fig. 4).]

Sur les figures 4 et 5 relatives à la nuance 6025 , nous avons précisé la dépendance en fréquence après recuit, respectivement :

- de la perméabilité initiale en géométrie en spirale,

- de la perméabilité relative maximum en géométrie en rondelles.

En toutes circonstances, la perméabilité décroît rapidement à partir de valeurs élevées quand la fréquence augmente. On retrouve un comportement 
comparable pour la perméabilité initiale en géométrie en rondelles et la perméabilité relative maximum en géométrie en spirale.

La nuance $2705 \mathrm{M}$ se comporte différemment ainsi qu'en témoignent les figures $6,7,8$ et 9 . Nous y avons précisé la dépendance en fréquence des perméabilités initiale et relative maximum pour les deux géométries. Il apparaît qu'un recuit sous champ transverse conduit dans les deux cas à des caractéristiques plates. Les courbes d'aimantation correspondantes, tracées sur la figure 10 pour la géométrie en rondelles, présentent une faible dépendance en fréquence et ont une allure linéaire jusqu'à un

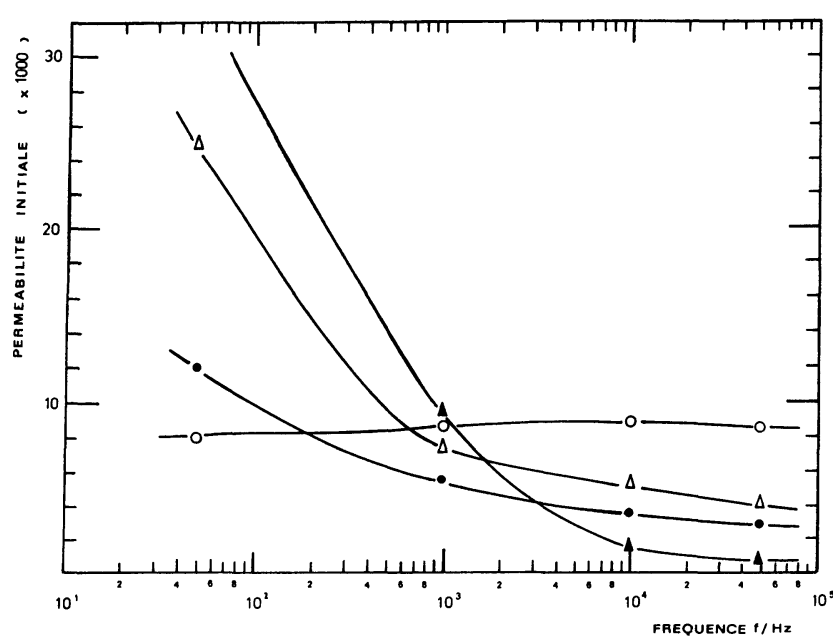

Fig. 6. - Dépendance en fréquence de la perméabilité initiale. - Alliage 2705 M. Géométrie en spirale (mêmes symboles que sur la Fig. 4).

[Initial permeability as a function of frequency. - Alloy 2705 M. Spiral geometry (symbols are those of Fig. 4).]

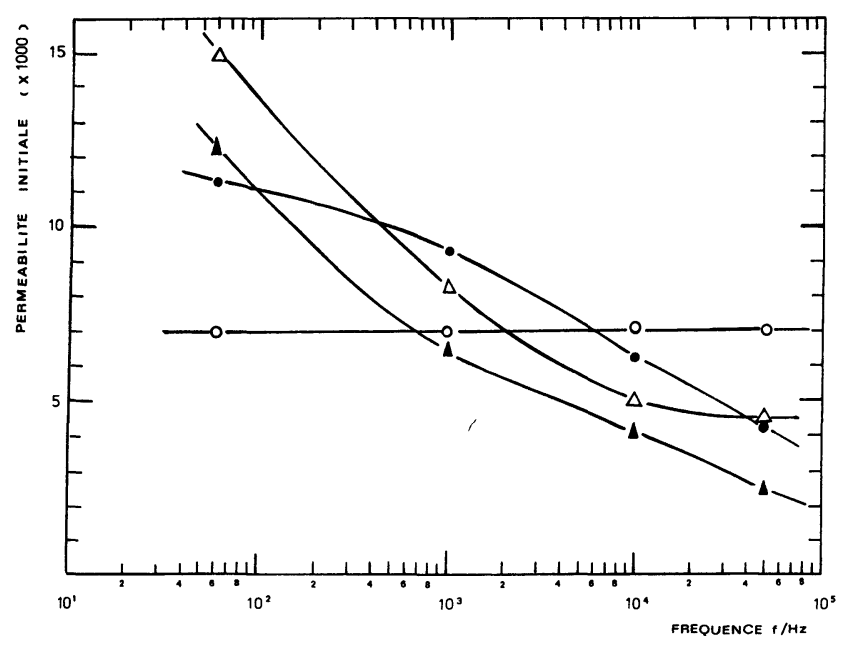

Fig. 7. - Dépendance en fréquence de la perméabilité initiale. - Alliage $2705 \mathrm{M}$. Géométrie en rondelles (mêmes symboles que sur la Fig. 4).

[Initial permeability as a function of frequency. - Alloy 2705 M. Ring geometry (symbols are those of Fig. 4).]

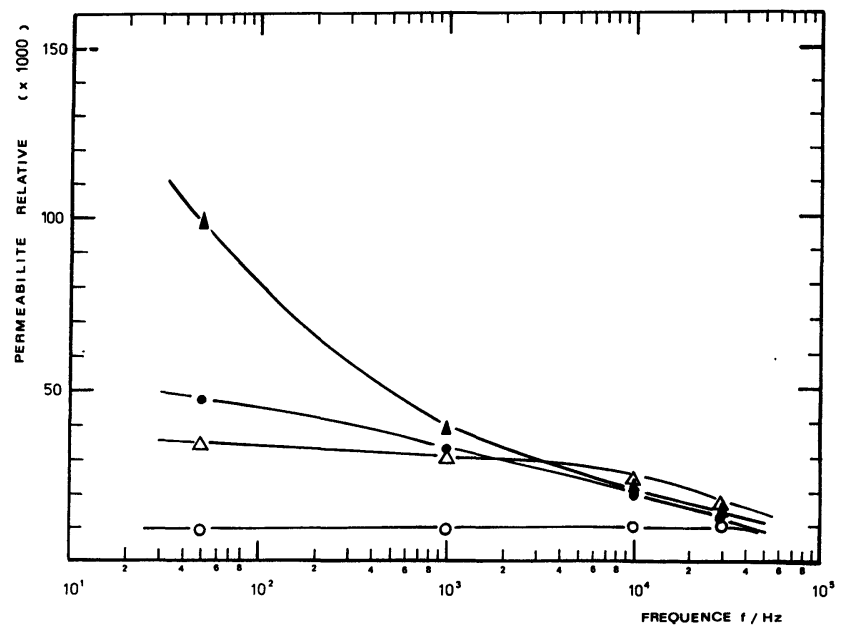

Fig. 8. - Dépendance en fréquence de la perméabilité relative maximum. - Alliage $2705 \mathrm{M}$. Géométrie en spirale (mêmes symboles que sur la Fig. 4).

[Maximum relative permeability as a function of frequency. - Alloy 2705 M. Spiral geometry (symbols are those of Fig. 4).]

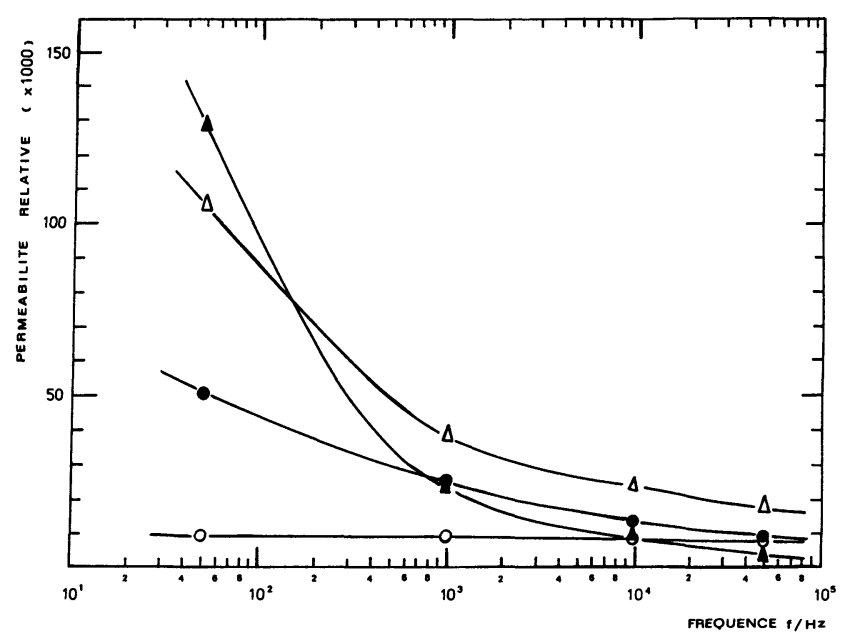

Fig. 9. - Dépendance en fréquence de la perméabilité relative maximum. - Alliage $2705 \mathrm{M}$. Géométrie en rondelles (mêmes symboles que sur la Fig. 4).

[Maximum relative permeability as a function of frequency. - Alloy $2705 \mathrm{M}$. Ring geometry (symbols are those of Fig. 4).]

niveau proche de la saturation. Il en est de même pour la géométrie en spirale. Ce comportement, favorable pour l'objectif envisagé, est spécifique du RACT.

Les pertes mesurées pour la nuance $2705 \mathrm{M}$ sont précisées sur le tableau III. Celles relatives au RACC appliqué à la géométrie en rondelles, très importantes, n'ont pas été reportées. Il apparaît que le RACT conduit aux valeurs les plus faibles. Ceci n'est pas vrai pour la nuance 6025 où RSC, RACC et RACT conduisent à des pertes comparables. 
Tableau III. - Pertes en W. $\mathrm{kg}^{-1}$ mesurées pour la nuance $2705 \mathrm{M}$.

[Measured losses (W. $\mathrm{kg}^{-1}$ ) of $2705 \mathrm{M}$ alloy.]

\begin{tabular}{|c|c|c|c|c|c|c|c|c|}
\hline \multirow{3}{*}{$\underset{(\mathrm{kHz})}{F}$} & \multirow{3}{*}{$\begin{array}{c}B \\
(\mathrm{~T})\end{array}$} & \multicolumn{7}{|c|}{ Géométrie } \\
\hline & & \multicolumn{3}{|c|}{ Rondelles } & \multicolumn{4}{|c|}{ Spirale } \\
\hline & & Brut & RSC & RACT & Brut & RSC & RACC & RACT \\
\hline \multirow{2}{*}{10} & 0,1 & 1,22 & 2,17 & 0,42 & 2,72 & 3,48 & 7,09 & 0,21 \\
\hline & 0,4 & 13,6 & 20,5 & 7,93 & 17,9 & 32,7 & 39,3 & 4,26 \\
\hline \multirow{2}{*}{50} & 0,1 & 13,3 & 14,9 & 2,12 & 7,76 & 20,2 & 82,3 & 3,26 \\
\hline & 0,4 & 152 & 175 & 71,8 & 139 & 272 & 336,8 & 51,1 \\
\hline
\end{tabular}

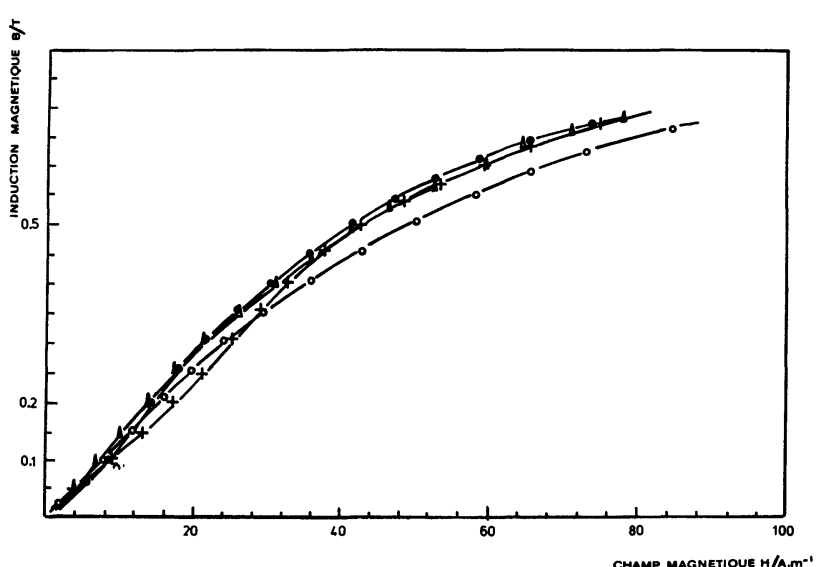

Fig. 10. - Courbes d'aimantation $B(H)$. - Alliage $2705 \mathrm{M}$. RACT. Géométrie en rondelles. $\bigcirc 50 \mathrm{~Hz}$, $\Delta 1 \mathrm{kHz}, \odot 10 \mathrm{kHz},+50 \mathrm{kHz}$.

[Magnetization curves $B(H)$. - Alloy 2705 M. Transversal field annealing. Ring geometry. $\bigcirc 50 \mathrm{~Hz}, \triangle 1 \mathrm{kHz}$, - $10 \mathrm{kHz},+50 \mathrm{kHz}$.]

\section{Discussion.}

Nous avons rassemblé sur la figure 11 trois photographies de la structure en domaines de l'alliage $2705 \mathrm{M}$. La technique opératoire met en œuvre l'effet Kerr.

La structure 11a est observée après un recuit sans champ. Les domaines sont relativement ordonnés et orientés obliquement par rapport à l'axe de l'échantillon. Il est donc fortement probable que le fabricant soumet ce type de ruban à un traitement magnétothermique avant commercialisation. L'observation des domaines sur les matériaux bruts confirme ce point de vue. Pour le 6025 , les domaines présentent une structure non ordonnée, finement divisée, tandis que pour le $2705 \mathrm{M}$, la structure est ordonnée, les domaines larges et orientés selon l'axe du ruban. Ainsi ce dernier alliage serait soumis avant commercialisation à un traitement magnétothermique de type circonférentiel.
Les photographies $11 \mathrm{~b}$ et $\mathrm{c}$ décrivent la structure magnétique après traitement, respectivement, sous champ circonférentiel et sous champ transverse. Elles suggèrent une interprétation qualitative des mesures de pertes.

Le recuit sous champ circonférentiel crée un axe de facile aimantation approximativement selon l'axe du ruban $\left({ }^{2}\right)$. Les domaines observés sont de grande taille et à $180^{\circ}$. Quand le ruban est soumis au champ de mesure, nous observons un déplacement des parois de Bloch parallèlement à elles-mêmes. Cette observation sous microscope est actuellement limitée aux basses fréquences. Nous avons, pour ce faire, mis en œuvre une méthode stroboscopique par hachage du faisceau laser. Aux basses frequences, ces déplacements ne provoquent pas une dépense importante d'énergie. Dans ces conditions, les perméabilités sont élevées (Figs. 6 et 7) et les cycles, rectangulaires. Aux fréquences élevées, les parois de grande longueur ne peuvent se déplacer facilement au rythme imposé sur des distances de l'ordre de plusieurs centaines de microns. Les pertes magnétiques sont importantes, le cycle d'hystérésis s'incline tandis que l'approche à la saturation nécessite un champ magnétisant notable.

Après traitement sous champ transverse (Fig. 11c), l'orientation des domaines est normale à l'axe du ruban. Ces domaines sont de trop grande taille pour tourner en bloc de façon à s'orienter selon le champ de mesure. Ils se divisent préalablement. Cette procédure conduit à des pertes plus élevées en basse fréquence que celles résultant des déplacements de parois (cas du champ circonférentiel). Aux hautes fréquences, la situation est inversée.

Nous avons observé cette division, toujours à basse fréquence. Si le champ appliqué durant la mesure est faible (inférieur à $1000 \mathrm{~A} \cdot \mathrm{m}^{-1}$ environ),

$\left({ }^{2}\right)$ L'intensité du champ circonférentiel appliqué était probablement insuffisante pour faire totalement disparaître l'anisotropie magnétique observée sur la figure 12a. 

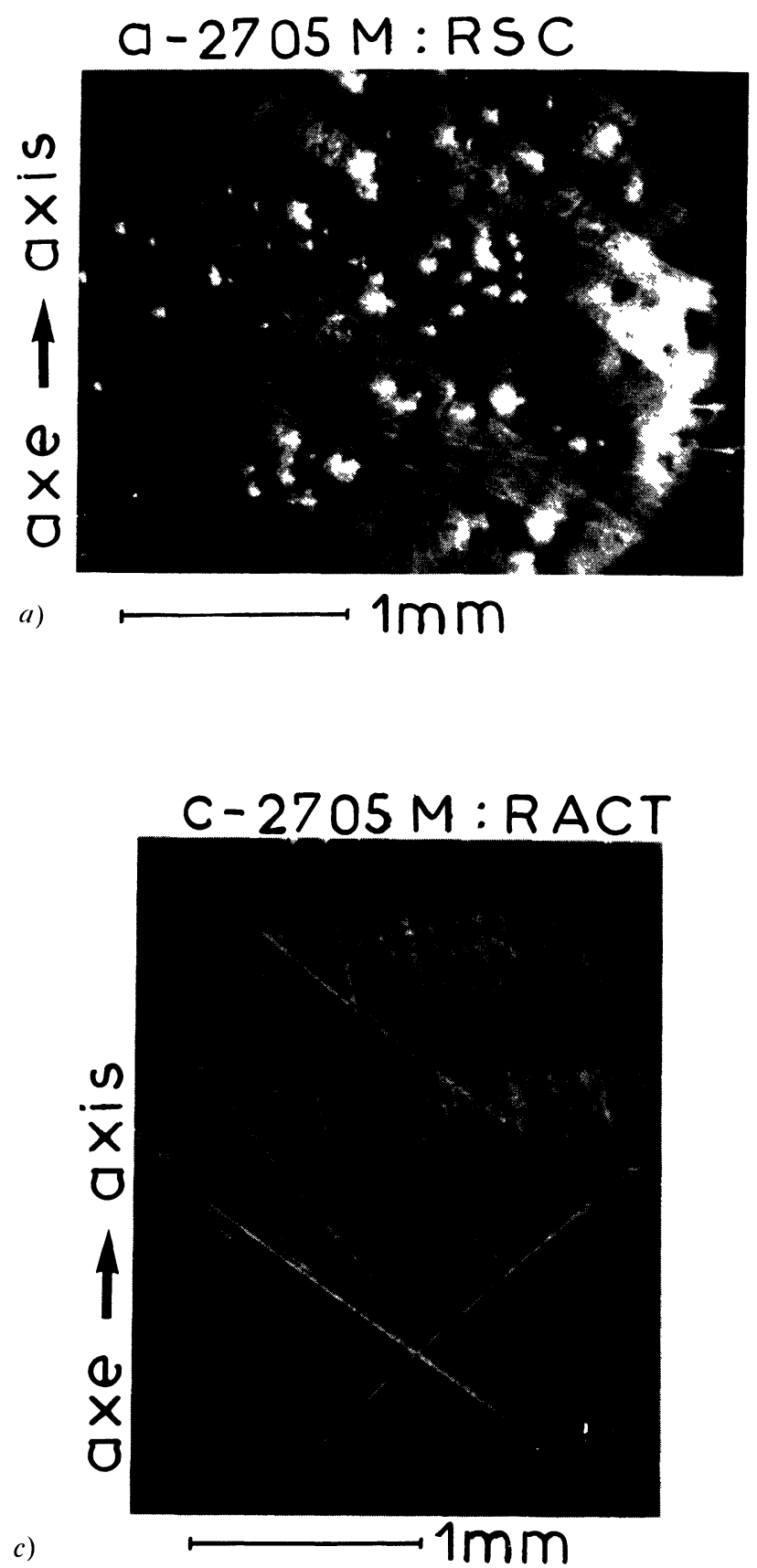

Fig. 11. - Structure en domaines observée sur l'alliage 2705 M, a - RSC, b - RACC, c - RACT.

[Domain structure observed on the alloy $2705 \mathrm{M}$, a Without Field Annealing, b - Circumferential Field Annealing, c - Transversal Field Annealing.]

les domaines triangulaires effilés apparaissant en clair dans l'axe des domaines sombres (Fig. 12), ou vice versa, se développent longitudinalement et séparent progressivement le domaine qui les enserre. Pour un champ appliqué intense, le processus de division est apériodique et ne se prête plus à une analyse stroboscopique. Néanmoins, l'observation ne laisse pas de doute sur la division des domaines.
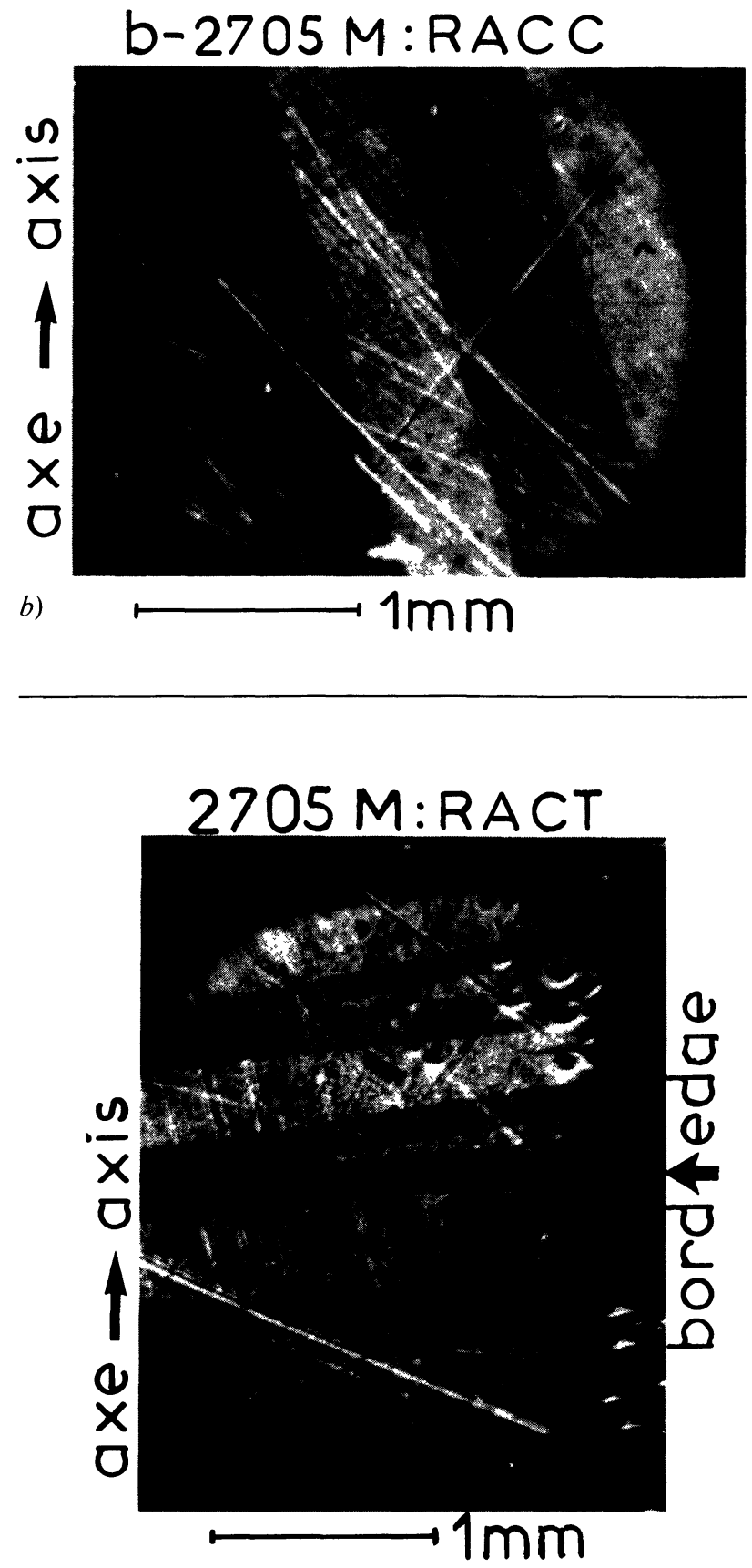

Fig. 12. - Initialisation de la division des domaines avant rotation à $90^{\circ} \mathrm{C}$.

[Beginning of the division process of the domains preceding the $90^{\circ}$ rotation.]

\section{Conclusion.}

Pour les deux géométries de tore, la nuance $2705 \mathrm{M}$ présente, après traitement sous champ transverse, et pour la bande de fréquences considérées $(50 \mathrm{~Hz}$ $50 \mathrm{kHz}$ ), une perméabilité pratiquement constante en fonction du champ appliqué et de la fréquence. Le cycle d'hystérésis est étroit et les pertes faibles. 
Ce matériau ainsi traité paraît donc intéressant pour la réalisation de têtes magnétiques analogiques.

Certains chercheurs, japonais notamment [9], estiment, preuves expérimentales à l'appui, que les alliages amorphes à base de cobalt présentent des propriétés magnétiques intéressantes dans la bande des fréquences vidéo (1-6 MHz). Aussi ce travail pourrait-il se prolonger par l'étude des propriétés magnétiques de ces matériaux jusqu'à de telles fréquences.

\section{Remerciements.}

Nous remercions la Société LCC-CICE du groupe Thomson pour sa contribution aussi bien scientifique que financière.

\section{Bibliographie}

[1] WANG, X. L. et al., IEEE Trans. Mag. 18 (1982) 1188. [5] Отомо, S. et al., in « Proc. 4th Int. Conf. on Rapidly [2] National Materials Advisory Board, « Report of the Committee on Magnetic Materials » (Publication NMAB-426, National Academy of Sciences. National Research Council, Washington, D.C. 1985).

[3] WHITE, R. M., Science 229 (1985) 11.

Quenched Metals » Sendai (1981) 1023.

[6] Hilzinger, H. R., Kanz, W., J. Magn. Mat. 15-18 (1980) 2480.

[7] O'Handley, R. C., Sullivan, M. O., J. Appl. Phys. 52 (1981) 1841.

[8] OChiaI, Y. et al., J. Appl. Phys. 55 (1984) 3033.

[9] SHIIKI, K. et al., J. Appl. Phys. 52 (1981) 2483. 